\title{
Fast analytical calculation of the air-gap flux density in an outer-rotor permanent-magnet brushless motor
}

\author{
Shuaichen $\mathrm{Ye}^{*}$, and Xiaoxian Yao \\ School of Aerospace Engineering, Beijing Institute of Technology, Beijing, China
}

\begin{abstract}
The air-gap flux density must be considered in the design and optimization of the structure parameters of permanent-magnet brushless motors (PMBMs). Existing methods for calculating the air-gap flux density are complex and thus cannot be easily applied in practical engineering. This paper presents a fast analytical method for calculating the air-gap flux density that is more efficient and practicable than existing methods. A lumped magnetic circuit model is presented to illustrate the proposed method. Then, the analytical result and finite element analysis (FEA) results obtained for a PMBM prototype are compared. The results indicate that the error between the two methods does not exceed 5\%. Therefore, the proposed analytical method is highly efficient and accurate, which may be applied in the motor pro-design process of many engineering instruments.
\end{abstract}

\section{Introduction}

Brush-type DC motors and cage induction motors have been widely used in electrical traction. However, these motors are not adequate for a directly gearless electromechanical driving system, as they can only drive the axis of the wheel with the aid of a conveyor belt or gear sets.

An outer-rotor permanent-magnet brushless motor (PMBM) can drive the load directly by fitting into a wheel that ideally fits the outer rotor. Such is the case for applications of traction in various types of equipment, such as wheelchairs, self-balancing unicycles and rotor wings in unmanned aerial vehicles (UAVs). A more cumbersome construction is to arrange the outer rotor with a flange, which can connect the wheel to the rotor and drive it [1].

The number of applications of PMBMs in industry has been increasing in recent years due to the many advantages of PMBMs over other types of electrical machines, including their high efficiency, large torque-to-mass ratio (also referred to as torque density), long operational life, good dynamic performance and quiet operation [2]. Therefore, research on the performance of this type of motor is important.

The performance of a PMBM is typically measured by its output torque and rotation speed, which are strongly related to the air-gap flux density. Thus, the air-gap flux density must be considered in the design and optimization of PMBMs. In recently relevant

\footnotetext{
*Corresponding author: yesc_bit@163.com
} 
literatures, Msaddek [3] analyzed two design and optimization methods for the outer-rotor PM motor using the air-gap density as an optimization variable. Feng and Zhang [4] proposed an analytical model for surface-mounted PM synchronous machines. This model was used to optimize the efficiency of the motor, with air-gap flux density considered as a constraint.

In the aforementioned studies, the air-gap flux density had to be determined. There are three approaches currently used to calculate the air-gap flux density: Maxwell theory, the finite element analysis (FEA) method (numerical method) and the analytical method. Boroujeni [5] proposed a method to calculate the air-gap flux density and cogging torque of PM machines based on Maxwell stress tensor theory. Kim [6] and Liu [7] presented FEA methods respectively to determine the air-gap flux density. Maxwell theory and the FEA method are relatively accurate but are often time consuming and complex.

Lubin [8] established an analytical model of magnetic circuits to compute the magnetic field distribution in PM motors. However, this analytical method can only be used when all of the structural parameters of the motor are known with accuracy. In some engineering designs, particularly pre-design processes, it is impossible to obtain all of the structural parameters. To eliminate this problem and increase the efficiency of the air-gap flux density calculation, this paper presents a fast analytical method to compute the air-gap flux density of a no-load outer-rotor PMBM.

\section{Analytical model for calculating the air-gap flux density}

In this paper, an outer-rotor PMBM with four poles and six slots is used to demonstrate the proposed approach. The simplified cross section of the motor is shown in figure 1 . The permanent magnet is composed of sintered $\mathrm{NdFeB}$, which has a high residual flux density $B_{r}$ and a high coercive force $H_{c}$. The demagnetization curve of $\mathrm{NdFeB}$ (figure 2) is straight.

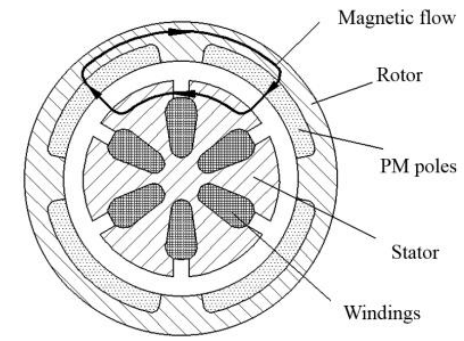

Fig. 1. Simplified cross section of the motor.

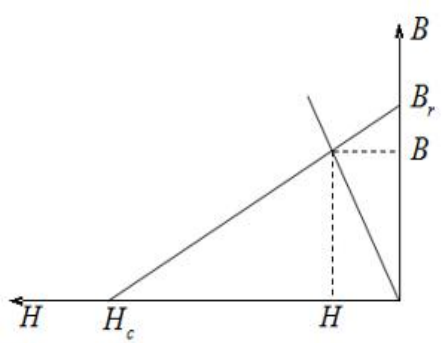

Fig. 2. Demagnetization curve of $\mathrm{NdFeB}$.

The equation of arbitrary point $(B, H)$ on this curve is:

$$
B=B_{r}-\frac{B_{r}}{H_{c}} H=B_{r}-\mu_{0} \mu_{r} H
$$

wherein $\mu_{0}$ is the air permeability and $\mu_{r}$ is the relative permeability of the magnet.

Thus, the magnetomotive force $F_{m}$ and flux $\Phi_{m}$ of the permanent magnet poles in motor can be represented as:

$$
\left\{\begin{array}{l}
\Phi_{m}=B S_{m} \\
F_{m}=H h_{m}
\end{array}\right.
$$

where $S_{m}$ is the magnet area perpendicular to the magnetizing direction and $h_{m}$ is the magnet thickness.

Substituting equation (1) into equation (2) yields:

$$
\Phi_{m}=B_{r} S_{m}-\mu_{0} \mu_{r} H S_{m}=B_{r} S_{m}-\frac{\mu_{0} \mu_{r} S_{m}}{h_{m}} H h_{m}
$$


Define $\Phi_{r}=B_{m} S_{m}$ as the intrinsic flux of the magnet pole and $R_{m}=h_{m} /\left(\mu_{0} \mu_{r} S_{m}\right)$ as the reluctance of the magnet pole. Consequently, the permanent magnet pole can be recognized as a flux source paralleling with a constant reluctance.

The equivalent magnetic circuit and simplified magnetic circuit model are shown in figure 3 and figure 4 respectively.

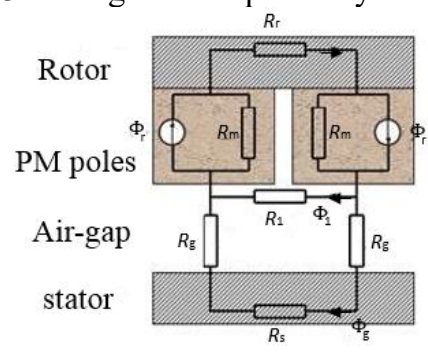

Fig. 3. Equivalent magnetic circuit of the PMBM.

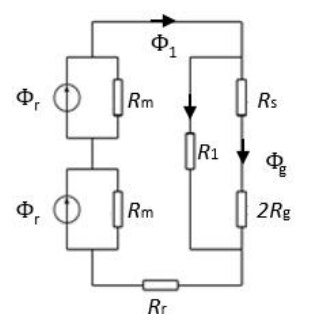

Fig. 4. Simplified magnetic circuit model.

According to the magnetic circuit theory:

$$
\begin{gathered}
\Phi_{1}=\frac{\frac{2 R_{m}}{\frac{2 R_{1} R_{s} R_{g}}{R_{1}+R_{s}+2 R_{g}}+R_{r}+2 R_{m}} \cdot 2 \Phi_{r}}{\Phi_{g}=\Phi_{1} \cdot \frac{R_{1}}{2 R_{g}+R_{s}+R_{1}}=2 \Phi_{r} \cdot \frac{2 R_{m} R_{1}}{2 R_{1} R_{s} R_{g}+\left(R_{r}+2 R_{m}\right) \cdot\left(2 R_{g}+R_{s}+R_{1}\right)}}
\end{gathered}
$$

where $R_{r}$ is the reluctance of the rotor core, $R_{s}$ is the reluctance of the stator core, $R_{1}$ is the reluctance due to the magnet-to-stator flux leakage, and $R_{\mathrm{g}}$ is the reluctance of the air-gap. $R_{\mathrm{g}}=\delta /\left(\mu_{0} A_{\mathrm{g}}\right)$, where $\delta$ is the air-gap length and $A_{\mathrm{g}}$ is the surface area of the air-gap. Then, the air-gap flux density can be determined as:

$$
B_{g}=4 B_{r} R_{m} R_{1} /\left[2 R_{1} R_{s} \delta / \mu_{0} A_{g}+\left(R_{r}+2 R_{m}\right) \cdot\left(2 \delta / \mu_{0} A_{g}+R_{s}+R_{1}\right)\right]
$$

$\alpha_{m}$ is the effective pole arc coefficient.

It should be mentioned that the proposed calculation does not account for the saturation of the stator core material. Actually, the flux density distribution of the entire motor is highly complex. The proposed method can only obtain the average air-gap flux density, which is adequate for the design and optimization of a PMBM.

\section{Structure and parameters of the PM motor prototype}

To verify the feasibility of the proposed analytical method, an 8-poles outer-rotor PM motor was considered as an example. The cross section of the motor is shown in figure 5 , and the motor's structural parameters are provided in Table 1. In this prototype, 8 pieces of PM (NdFeB) are mounted alternately in the inner surface of the rotor, and two adjacent PMs comprise a pair of poles. The pole pitch can be extremely short with a large number of poles, and this multipole magnetic arrangement reduces the magnetic iron yoke.

The stator core and rotor core are laminated by $0.35 \mathrm{~mm}$-thick silicon steel. The poles are composed of sintered NdFeB (supplied by JSOL Corporation, Japan), and characteristic parameters of the sintered $\mathrm{NdFeB}$ are provided in Table 2.

The distribution of stator windings and external circuit of this prototype are shown in figure 6 . The windings use three-phase $\mathrm{Y}$ connections and the coil span is designed to be equal to the pole pitch. The external circuit is a three-phase bridge inverter circuit with 6 states. Additional controller and feedback devices should be integrated into the system to contribute to a complete PWM control circuit. The final PWM control circuit can ensure that only two phases conduct at any given time. 


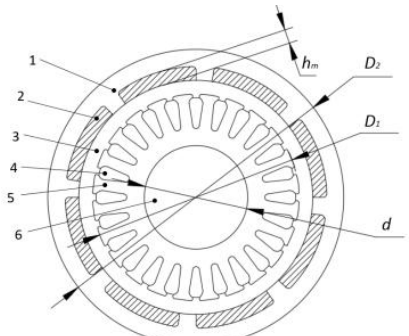

1 rotor 2 PM poles 3 air-gap 4 windings 5 stator 6 shaft

Fig. 5. Cross section of the outer-rotor PM motor.

Table 1. Specifications of the motor.

\begin{tabular}{|l|l|l|}
\hline \multicolumn{1}{|c|}{ Parameters } & Value & Unit \\
\hline Motor type & 3 phase $8 \mathrm{p} / 24 \mathrm{~s}$ & - \\
\hline Rate speed & 3000 & $\mathrm{rpm}$ \\
\hline Driving voltage & 24 & $\mathrm{~V}$ \\
\hline Outer diameter of stator core $\left(\mathrm{D}_{1}\right)$ & 30 & $\mathrm{~mm}$ \\
\hline Outer diameter of rotor core $\left(\mathrm{D}_{2}\right)$ & 43 & $\mathrm{~mm}$ \\
\hline Air-gap length $(\delta)$ & 1.5 & $\mathrm{~mm}$ \\
\hline Axial length $(\mathrm{L})$ & 40 & $\mathrm{~mm}$ \\
\hline PM thickness $\left(h_{m}\right)$ & 2.5 & $\mathrm{~mm}$ \\
\hline Diameter of shaft $(\mathrm{d})$ & 15 & $\mathrm{~mm}$ \\
\hline
\end{tabular}

Table 2. Specifications of the permanent magnet poles.

\begin{tabular}{|l|l|l|}
\hline \multicolumn{1}{|c|}{ Parameters } & Value & Unit \\
\hline Residual flux density $B_{r}$ & $1.17-1.22$ & $\mathrm{~T}$ \\
\hline Coercivity force $H_{c}$ & $\geq 844$ & $\mathrm{KA} / \mathrm{m}$ \\
\hline Intrinsic coercivity force $H_{c}$ & $\geq 1592$ & $\mathrm{KA} / \mathrm{m}$ \\
\hline (BH)max & $247-271$ & $\mathrm{KJ} / \mathrm{m}^{3}$ \\
\hline Density $\rho$ & $7500-7700$ & $\mathrm{Kg} / \mathrm{m}^{3}$ \\
\hline
\end{tabular}

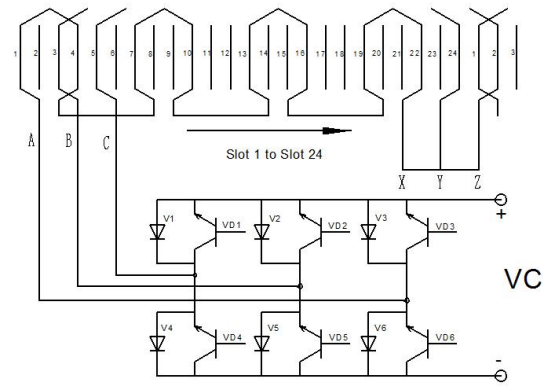

Fig. 6. Winding distribution and external circuit.

\section{Results and discussion}

\subsection{Basic mathematical formulation}

The 2-D differential partial equation of the outer-rotor PMBM expressed by magnetic vector potential $\vec{A}$ is:

$$
\frac{\partial}{\partial x}\left(\frac{1}{\mu_{r}} \frac{\partial \vec{A}}{\partial x}\right)+\frac{\partial}{\partial y}\left(\frac{1}{\mu_{r}} \frac{\partial \vec{A}}{\partial y}\right)=-\frac{N}{s}\left(I_{X}+I_{Y}+I_{Z}\right)-\frac{1}{\mu_{r}}\left(\frac{\partial(B H)_{y}}{\partial x} \frac{\partial(B H)}{\partial y}+\phi \frac{\partial \vec{A}}{\partial t}+\nabla \Phi\right)
$$


Because the divergence of the magnetic field is equal to zero, the magnetic vector potential $\vec{A}$ is defined as:

$$
\nabla \cdot \vec{B}=\nabla \cdot(\nabla \times \vec{A}) \equiv 0
$$

In equation (7), $\mu_{r}$ is the permeability of the permanent magnet, $\mathrm{N}$ is coil turns of each phase, $\mathrm{s}$ is the approximate cross-sectional area of each slot, $\mathrm{X}, \mathrm{Y}$, and $\mathrm{Z}$ represent each phase, and $\mathrm{x}, \mathrm{y}$ are the two dimensions.

In this research, the eddy current densities $\mathrm{J}$ of the stator and rotor were neglected. Therefore, the last item in equation (7) can be expressed as:

$$
\nabla J=\nabla \sigma\left(-\frac{\partial \vec{A}}{\partial t}-\nabla \Phi\right)=0
$$

wherein $\sigma$ is the electric conductivity.

After discretization equation (7) can be expressed in finite element form as well as in terms of the Galerkin equation.

\subsection{Analytical and FEA results}

Next, the proposed analytical method was applied to the above-defined motor. The flux leakage was neglected, and the stator core reluctance $R_{S}$ and rotor core reluctance $R_{r}$ were defined as 0.01 times the air-gap reluctance $R_{\mathrm{g}}$. The average air-gap flux density can be calculated as $0.98 \mathrm{~T}$, and its approximate trapezoidal waveform is shown in figure 7.

To verify the accuracy of the analytical results, the air-gap flux density was calculated via FEA using JMAG software. JMAG is a powerful and practical motor-simulation software developed in Japan. The main purpose of JMAG is to perform FEA. The flux contour plot (figure 8) and air-gap flux density (figure 9) of the motor can be obtained after performing the modeling, setting the parameters and performing the meshing processes. The simulation indicated that the analytical results were largely consistent with the FEA results. Furthermore, the average air-gap flux density from JMAG was $1.01 \mathrm{~T}$ which indicated that the errors between the FEA and analytical results were less than 5\%.
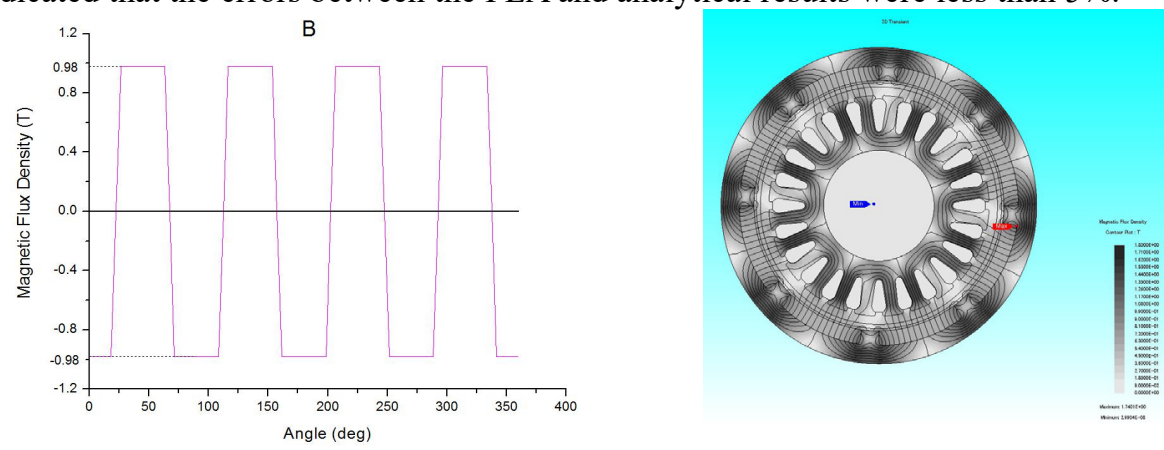

Fig. 7. The approximate trapezoidal waveform Fig. 8. The flux contour plot of motor. of air-gap flux density.

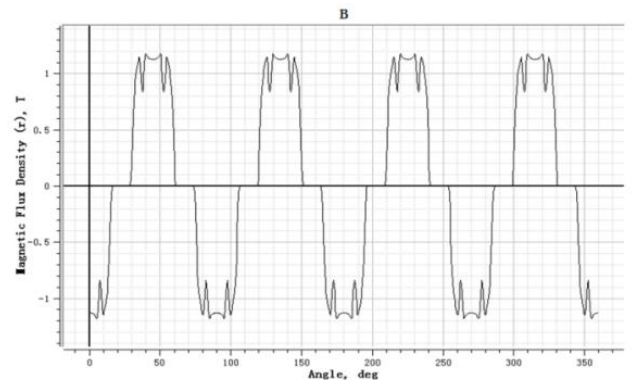

Fig. 9. Air-gap flux density calculated by the FEA method. 


\subsection{Discussion}

The proposed analytical method is faster than the FEA method, and it also suitable for predicting the air-gap flux density of a PMBM when the machine structure parameters are not precisely known. Furthermore, the results demonstrate that this method is fairly accurate. The approach outlined in this study can be applied in the motor pro-design process of many engineering instruments.

However, this method is only appropriate for the no-load situation. Under a load condition, the armature reaction and saturation of the core should be considered. In future studies, an extended model that consider the load for optimizing the out-put torque of the PMBM should be established.

\section{Conclusion}

The novel fast and accurate 2-D no-load analytical method for calculating the average airgap density of a PMBM is the primary contribution of this paper. The results demonstrate that there is only a 5\% error between the analytical and FEA results for different machine structure parameters. Thus, the analytical method can be used to calculate the air-gap flux density of PMBMs directly.

\section{References}

1. López-Fernandez X M, Gyselinck J and Silveria-Correa R 2006 Finite element analysis of an outer-rotor permanent-magnet brushless DC motor for light traction COMPEL 25 (3) $\mathrm{pp}$ 705-12

2. Knypiñski L and Nowak L 2013 Optimization of the permanent magnet brushless DC motor employing finite element method COMPEl 32(4) pp 1189-202

3. Msaddek H, Brisset S, Mansouri A and Trabelsi H 2015 Design and optimization of PMSM with outer rotor for electric vehicle IEEE 12th International Multi-Conf. on Systems, Signals \& Devices (SSD15) pp 1-6

4. Feng Y L and Zhang C N 2017 Analytical calculation for predicting the air gap flux density of surface-mounted permanent magnet synchronous machine J. Electr Eng Technol. 12(2) pp 769-77

5. Boroujeni S T and Zamani V 2015 A Novel Analytical Model for No-Load, Slotted, Surface-Mounted PM Machines: Air Gap Flux Density and Cogging Torque IEEE Trans. Magn. 51(4) 8104008

6. Kim J S, Lee J H, Song J Y, Kim D W, Kim Y J and Jung S Y 2017 Characteristics Analysis Method of Axial Flux Permanent Magnet Motor based on Two-Dimensional Finite Element Analysis IEEE Trans. Magn. 53(6) 8105304

7. Lin M J, Han Z,Pei Y W, Shi P F 2013 Optimization of Permanent Magnet Motor Airgap Flux Density Based on the Non-uniform Air Gap Proc. 2013 International Conference on Mechatronic Sciences, Electric Engineering and Computer (MEC) pp 3422-26

8. Lubin T, Mezani S and Rezzoug A 2011 2-D exact analytical model for surfacemounted permanent-magnet motors with semi-closed slots IEEE Trans. Magn. 47(2) pp 479-92 